\title{
Le quidditch moldu
}

De l'imaginaire à la réalité

Muggle quidditch. From Fantasy to Reality

\section{Audrey Tuaillon Demésy}

\section{OpenEdition}

\section{Journals}

Édition électronique

URL : https://journals.openedition.org/questionsdecommunication/11208

DOI : 10.4000/questionsdecommunication. 11208

ISSN : 2259-8901

\section{Éditeur}

Presses universitaires de Lorraine

\section{Édition imprimée}

Date de publication : 1 septembre 2017

Pagination : 393-413

ISBN : 9782814303256

ISSN : 1633-5961

\section{Référence électronique}

Audrey Tuaillon Demésy, « Le quidditch moldu », Questions de communication [En ligne], 31 | 2017, mis en ligne le 01 septembre 2019, consulté le 26 octobre 2021. URL : http://journals.openedition.org/ questionsdecommunication/11208; DOI : https://doi.org/10.4000/questionsdecommunication. 11208 


\section{AUDREY TUAILLON DEMÉSY}

Culture, sport, santé, société

Université de Franche-Comté

F-25000

audrey.tuaillon-demesy@univ-fcomte.fr

\section{LE QUIDDITCH MOLDU DE L'IMAGINAIRE À LA RÉALITÉ}

Résumé. - Le quidditch est une pratique physique issue de l'univers fictionnel Harry Potter. Adaptant une activité provenant d'un monde merveilleux, les joueurs se réapproprient un imaginaire et participent à la (re)création de ce sport. L'objectif de cet article est de proposer, à l'aide d'une méthodologie socio-ethnographique (observations et entretiens), une lecture du quidditch sous l'angle de la construction de monde(s), en interrogeant le passage de l'imaginaire à la réalité. Au-delà, la mise en vie du quidditch invite à saisir les logiques transmédiatiques cristallisant le passage d'un monde à l'autre, ainsi que les processus à l'œuvre permettant l'émergence d'une communauté de pratique.

Mots clés. - communauté de pratique, construction de mondes, Fantasy, fiction, quidditch, re-création, transmédia, worlds-building 
$\mathrm{P}$ ar un matin brumeux et froid de février, le long d'une pelouse marquée d'un tracé ovale, arrivent peu à peu de jeunes adultes portant sacs de sports, bâtons en plastique, crampons et bandeaux sur la tête. Les équipes se regroupent près du tableau d'affichage, sur lequel sont répertoriés les matchs à venir. À côté du planning, un mémo jaune, sur lequel l'on peut lire : « The snitch was here », littéralement, « Le Vif était ici ». Ces petites feuilles amovibles se retrouveront plus tard sur les maillots des joueurs, les sacs, d'autres panneaux, etc. Les équipes se mettent en place sur les trois terrains disponibles et les joueurs commencent leur échauffement, arborant des tenues représentant leur appartenance à une équipe : les Paris Frog sont en vert, les Crookshanks de Lyon en rouge et bleu, les Black Snitches en noir, etc. Les animaux occupent une place centrale dans les logos des équipes : grenouille, chat, éléphant, hermine et chouette se disputent les honneurs. Une fois l'échauffement achevé, les ballons sont positionnés au centre du terrain dans un ordre précis : deux cognards, un souafle et, de nouveau, un cognard. L'arbitre annonce alors « Balais à terre! ». Les joueurs se positionnent, bâton en main, mais posé au sol. Le match est officiellement lancé par un « Brooms' up! » de l'arbitre : les joueurs montent sur leurs « balais » et se précipitent en milieu de terrain pour se saisir des balles. Pendant dix-huit minutes, ils vont s'affronter : les trois poursuiveurs cherchent à se saisir du souafle pour l'envoyer dans l'un des trois anneaux de l'équipe adverse, tandis que les deux batteurs de chaque groupe, à l'aide des cognards, tentent de mettre hors-jeu leurs adversaires. Quant aux gardiens, ils s'interposent devant les cerceaux, afin d'empêcher le souafle de traverser. Sur le bord, les joueurs remplaçants encouragent ceux qui sont sur le terrain et attendent de pouvoir y entrer. Arrive alors le porteur du Vif d'or - entièrement vêtu de jaune -, suivi des attrapeurs. Ceux-ci s'opposent pour récupérer une balle de tennis (le vif proprement dit), accrochée au dos de son porteur. Quelques minutes plus tard, elle est attrapée, ce qui met fin à la partie. Les joueurs s'embrassent et retournent sur la zone d'accueil afin de prendre connaissance des différents résultats. Durant un week-end complet', les matchs s'enchaînent. Finalement, la finale verra s'opposer deux équipes parisiennes et les Titans remporteront la victoire. Outre les supporteurs incontestés, quelques dizaines de spectateurs sont présents, simples promeneurs, fans ou curieux. Ils s'étonnent, en premier lieu, de ne pas voir de « vrais » balais, symbolisant le monde d'Harry Potter ${ }^{2}$ et représentant l'outil incontournable de la pratique du quidditch. À la place de balais, en effet, des bâtons gris en PVc, dont les bouts ont été protégés à l'aide de rembourrage et de scotch. Cette transformation de l'objet-symbole de l'univers magique cristallise le passage d'un sport « sorcier » au quidditch moldu (terme inventé par J. K. Rowling pour désigner une pratique destinée à ceux qui n'ont pas de pouvoirs).

\footnotetext{
' Cette description introductive est issue de la seconde Coupe de France organisée à Toulouse en février 2016.

2 Lorsqu'il sera fait référence à l'ensemble de l'œuvre (romans et films), Harry Potter sera mis en italique ; autrement, le terme désignera le personnage.
} 
Cette activité dénommée tout simplement quidditch par les joueurs, est une pratique physique et sportive issue de la fiction ${ }^{3}$ Harry Potter. Déjà bien implanté dans le champ du sport universitaire aux États-Unis et en pleine expansion en Europe, le quidditch comprend aujourd'hui une quarantaine d'associations en France et ses adeptes peuvent se rencontrer lors de différents championnats et rencontres sur le territoire métropolitain ${ }^{4}$. D'autres manifestations regroupent les meilleures équipes françaises à l'étranger : Coupes du monde, d'Europe (par club et par nation), tournois amicaux, etc. Le quidditch moldu est une adaptation du quidditch magique et se présente comme une re-création d'une pratique physique (Tuaillon Demésy, 20 16) car il prend, au départ, ses racines dans un univers fictionnel. II est ainsi créé « de nouveau » dans un cadre « réel », non imaginaire. En prolongeant la fiction et en complétant le monde d'Harry Potter, ce quidditch se détache également de son homonyme magique. Les joueurs se réapproprient l'activité pour qu'elle devienne autonome, indépendante de l'univers sorcier. L'objectif de cet article est de montrer comment une pratique physique peut se situer à l'interface entre la construction d'un monde immersif (auquel fait référence le terme anglais worldbuilding) et l'édification d'un monde social (Becker, 1982), voire d'une communauté. Plus spécifiquement, il s'agit de réfléchir aux processus à l'œuvre lors de la mise en vie de cette activité, interrogeant le passage de l'imaginaire au monde sensible. La question est ainsi posée de savoir comment les logiques d'action à l'œuvre dans le monde fictionnel d'Harry Potter transparaissent dans les adaptations de la pratique physique du quidditch et structurent l'expérience des joueurs. Dans cet ordre d'idées, un double mouvement se laisse deviner : le processus de convergence culturelle expose un développement transmédiatique « classique » pour l'univers sorcier en tant que phénomène culturel et social, tandis que le quidditch moldu fait référence à une logique d'adaptation du monde magique.

L'étude proposée ici s'appuie sur un travail de terrain pluridisciplinaire, mené selon une approche socio-ethnologique. L'ensemble des données sur lesquelles repose cet article ont été recueillies à partir d'observations directes mises en place lors de différentes manifestations (Coupes de France en 2016 à Toulouse et Nantes, Coupe du monde à Francfort en juillet de la même année et entraînements associatifs), et d'entretiens semi-directifs réalisés avec des joueurs de quidditch français et étrangers, ainsi qu'avec des responsables de la fédération nationale. Plus précisément, une quarantaine de personnes ont pu être rencontrées et des liens privilégiés ont été tissés, en particulier avec l'une des associations de la fédération. Cette méthode de type ethnographique facilite la connaissance des modes d'actions en jeu au sein de la pratique, ainsi que des comportements des joueurs. Elle permet également de susciter l'intérêt et la confiance des pratiquants et de s'exposer, en tant que chercheur, à des confidences qui aident, par la suite, à saisir le fonctionnement et la genèse du groupe. En effet, les joueurs de quidditch

\footnotetext{
3 II s'agit ici des ouvrages écrits par J.K. Rowling (1997-2007) et de ses adaptations cinématographiques (200I-20I I).

${ }^{4}$ Voir note I.
} 
(quelques centaines en France) se reconnaissent comme faisant partie d'une même communauté, partageant valeurs et savoir-être communs, dont certains proviennent directement des thèmes abordés par les romans.

\section{Le monde magique : de la consommation à la participation}

\section{Harry Potter : phénomène culturel et social}

Le « phénomène » Harry Potter, tel que baptisé par les médias et divers spécialistes de l'édition ${ }^{5}$, englobe à la fois la production littéraire de J. K. Rowling (faisant ainsi référence au succès éditorial) et ses déclinaisons sur grand écran. Situées sur le podium des livres les plus vendus à l'échelle mondiale (et traduites dans plus de soixante langues), les aventures du jeune sorcier que chacun connait, au moins de nom, ont permis de générer la vente de plus de 400 millions d'ouvrages. Quant aux adaptations cinématographiques (huit films au total), elles s'élèvent à plus de sept milliards de recettes ${ }^{6}$.

Roman d'apprentissage et d'aventure ancré dans un univers fictionnel, le succès de cette production s'explique par les thématiques abordées (même si Harry Potter n'est pas la première œuvre de fantasy à mettre en scène magie et sorcellerie), mais surtout grâce à son ancrage dans la modernité (Smadja, 2007). En effet, si ces histoires se présentent comme des contes, elles ont pour particularité de s'adresser aux enfants d'aujourd'hui et de replacer le merveilleux dans le contexte du $x x^{e}{ }^{e}$ siècle. Ainsi, « Harry Potter est un conte de fées moderne » (Smadja, 200 I : 26). La magie est humanisée et permet une identification aisée des lecteurs à l'univers présenté. En outre, les romans, d'abord destinés à la jeunesse, ont trouvé, parmi les adultes, un public réceptif (Smadja, 2007).

Plus encore, ces écrits favorisent une réappropriation de l'univers par ceux qui sont devenus des experts de l'œuvre, des fans (Peyron, 2008). À cet égard, la saga a engendré une participation massive de la part des lecteurs, puisque les écrits de J. K. Rowling sont ceux bénéficiant le plus de production de fanfictions, ces récits prolongeant, modifiant ou complétant l'histoire originelle (François, 2009a). Dans la logique des fan studies, il est clair que la réception des œuvres de J. K. Rowling ne

\footnotetext{
${ }^{5}$ Voir, par exemple : Koninck (200I) ; ou encore, cette interview de J. K. Rowling (sur le site de l'INA) : http:// fresques.ina.fr/europe-des-cultures-fr/fiche-media/Europe00287/le-phenomene-harry-potter.html.

${ }^{6}$ Au sujet des adaptations voir notamment différents chiffres : http://www.telerama.fr/sortir/ harry-potter-la-saga-culte-en- I0-chiffres-cles, I 24946.php ; http://www.lemonde.fr/les-decodeurs/ article/20 I 6/06/04/en-livres-en-films-et-bientot-au-theatre-le-succes-de-harry-potter-enchiffres_4935392_4355770.html ; http://www.gazette-du-sorcier.com/Ventes-de-Harry-Potterquelques,892.
} 
s'est pas limitée à une lecture passive mais a engendré une « attitude active » (ibid. : 164). Concernant Harry Potter, ce phénomène est baptisé « Pottermania » par les lecteurs ; il fait état d'une forte réappropriation de l'histoire par les fans, permise par l'ancrage fictionnel du récit et par la spécificité éditoriale dont il a bénéficié (notamment concernant de nouvelles stratégies de ventes) (Raoul, 2007). Le désir de continuer à faire vivre le monde d'Harry Potter se décline autant dans les fanfictions que par le biais de sites internet dédiés, d'échanges numériques ou réels (à travers les conventions), de création de recettes de cuisines « magiques », etc. En ce sens, la Pottermania prend ses racines dans un contexte particulier qui laisse une place centrale aux interactions entre fans, mais aussi avec l'auteur, à travers les réseaux sociaux. L'univers d'Harry Potter a ainsi engendré des communautés de fans (Jenkins, 2006 ; François, 2009a), composées de passionnés qui se retrouvent autour d'objets, de supports et de fictions communes.

Ces communautés s'exposent à travers diverses plates-formes numériques ${ }^{7}$, proposant des discussions (tant sur l'histoire elle-même que sur des sujets variés liés au phénomène Harry Potter), des jeux, la possibilité de suivre des cours de magie, etc. L'accent est mis sur le fait de « jouer » un rôle, mais aussi sur la nécessité de proposer des espaces de dialogues. Ces lieux d'expression visent à faire participer les fans au monde de la fiction, à faire en sorte que la « magie » continue, autrement dit que le plaisir trouvé dans la lecture puisse être prolongé. En somme, un univers culturel (Donnat, 2004) est créé dans lequel les supports sont multiples, dépendant autant de l'auteur (films, livres) que de la communauté. Par extension, la reconnaissance des fans entre eux passe par un ensemble de traits culturels qui induisent une attitude et des manières de s'approprier un univers tourné vers la fantasy, le merveilleux, le fictionnel : « Être fan, comme geek, est une attitude générale qui n'est pas limitée à un support ou à un objet mais est liée à un rapport au monde et à un répertoire culturel » (Peyron, 20।4 : 57). La production médiatique (entendue ici au sens d'une pluralité de supports pour une même œuvre) autour d'Harry Potter a bénéficié de l'attitude active d'une partie de ses consommateurs. Ceux-ci ont, en effet, joué un rôle majeur dans le développement de la franchise et ont concouru à son succès grâce à leur participation. En ce sens, à l'image d'autres réalisations (notamment Star Wars ou Matrix, [Jenkins, 2006]), le succès d'Harry Potter repose sur le microcosme créé par l'auteur, devenu une référence incontournable de la culture populaire contemporaine.

\section{Univers sorcier et culture de la convergence}

Le poids culturel occupé aujourd'hui par cet univers fictionnel est lié à la pluralité des supports médiatiques sur lesquels s'expose le monde sorcier. En effet, Harry Potter est non seulement une série d'ouvrages, de films, de récits de fans, mais

\footnotetext{
${ }^{7}$ Des sites internet tels poudlard.org ou pottermore.com (tenu par J. K. Rowling elle-même) sont des exemples de plates-formes numériques qui réunissent les communautés de fans.
} 
l'histoire se décline aussi en jeux vidéo, en produits dérivés (jouets, vêtements, etc.), en pièce de théâtre, en expositions, en jeux de plateau et, bien sûr, sous la forme de re-créations, en particulier à travers le quidditch. Cette activité physique est issue d'un ouvrage écrit par J. K. Rowling (200 la, 200 I b) ${ }^{8}$ décrivant ce sport, son histoire, ses champions, etc. Cet essaimage de l'univers premier fait directement écho à la culture de la convergence, théorisée par Henry Jenkins. Orientée du point de vue des consommateurs, cette notion consiste à penser conjointement la « convergence médiatique », la « culture participative » et « l'intelligence collective » (Jenkins, 2006 : 22). Plus précisément, Henry Jenkins la définit comme le « flux de contenu passant par de multiples plateformes médiatiques, la coopération entre une multitude d'industries médiatiques et le comportement migrateur des publics des médias [...]. [Elle] est un mot qui permet de décrire les évolutions technologiques, industrielles, culturelles et sociales [...] » (ibid.). Dans cette logique, les éléments cinématographiques viennent compléter, par le biais d'une mise en scène visuelle, ce qui prend au départ une forme écrite (les ouvrages de J. K. Rowling).

Dès lors, la participation des fans modifie en profondeur l'ordre culturel habituellement établi puisque la production de la fiction n'est plus limitée aux grandes entreprises médiatiques mais se décline au niveau des individus et des communautés ${ }^{9}$. Le quidditch illustre parfaitement ce passage d'une description à une représentation d'une pratique physique qui n'existe pas en tant que telle dans le monde sensible. Répondant à l'« art de fabriquer des mondes » (Jenkins, 2006 : 42), le récit transmédiatique incite les consommateurs à recueillir des informations (sur différents supports médiatiques) pour compléter les connaissances qu'ils ont de l'univers. Chaque support permet ainsi de prolonger I'histoire (Peyron, 2016) et les pratiquants de quidditch moldu se retrouvent avant tout dans une attitude culturelle en lien avec l'univers d'Harry Potter. En ce sens, il est possible de faire l'hypothèse que la re-création du quidditch fait suite à cette recherche d'un monde fictif riche : en allant plus loin que ce qui est proposé par les industries, en se réappropriant l'activité et en lui donnant une nouvelle identité, les quiddkids ${ }^{10}$ contribuent à enrichir le monde d'Harry Potter et à dépasser le stade du récit pour entrer dans celui de l'action. La participation dépasse le stade de la consommation puisque les pratiquants de quidditch font

\footnotetext{
${ }^{8}$ Quidditch Through the Ages a été rédigé sous le pseudonyme de Kennilworthy Whisp, présenté comme un « expert renommé en matière de Quidditch ». L'objectif poursuivi ici est d'inscrire ce livre comme une composante à part entière du monde fictif. Ce n'est donc pas J. K. Rowling qui raconte l'histoire du quidditch, mais un historien issu de l'univers d'Harry Potter. On retrouve ici le principe d'« effet de réel » (Peyron, $2008: 357)$.

${ }^{9}$ Cette approche des œurres n'est pas sans poser quelques problèmes. Le phénomène des « Batailles de Potter » traduit certaines de ces difficultés, révélant des tensions en jeu entre producteurs et consommateurs/fans. Pour plus de compléments à propos des conflits entourant la parution des œuvres de J. K. Rowling, voir notamment H. Jenkins (2006, chap. 5).

${ }^{10}$ Quiddkids est le nom que les pratiquants de quidditch se donnent entre eux. Certains lui préfèrent « joueurs de quidditch », le terme quiddkids étant, selon eux, trop connoté. En français, il renverrait au domaine de l'enfance et de l'amusement.
} 
évoluer le monde fictionnel pour proposer une nouvelle activité ' ', oscillant entre une logique sportive (la pratique se spécialise, se normalise et s'institutionnalise [Guttmann, 1978]) et une approche en termes de culture geek'2 (Peyron, 20 I6).

Dans la lignée de la culture de la convergence, l'appropriation de ce sport révèle le rôle des acteurs (François, 2009b) dans les processus culturels, de même qu'une réception qui devient collective, davantage qu'individuelle (Jenkins, 2006). Par extension, le succès grandissant du quidditch s'explique également par la possibilité qu'il donne aux fans, notamment, de poursuivre l'expérience Harry Potter, sans qu'il y ait répétition avec d'autres offres. Dans cet ordre d'idées, le quidditch est davantage une re-création qu'un prolongement. Les fans sont producteurs de contenu, qu'il s'agisse de récits ou de pratiques ludiques et physiques. Le désir de re-créer le sport fétiche de leur héros s'inscrit dans la logique des « franchises » et répond parfaitement aux tendances contemporaines en termes de réappropriation par les consommateurs de biens culturels produits et/ou véhiculés par les industries médiatiques.

Pour autant, la construction d'un monde ne se limite pas à la diffusion d'une ligne narrative sur différents supports ${ }^{13}$. Dans la continuité des travaux menés par Lisbeth Klastrup et Susana Tosca (2004), ce qui importe sont les « propriétés » de ces « mondes transmédiaux » qui peuvent enclencher une re-création. Autrement dit, à partir du moment où les fans souhaitent prolonger la fiction en l'adaptant pour pouvoir la « vivre » (physiquement parlant), le quidditch devient une expérience partagée. En outre, il répond à des adaptations techniques (les joueurs, comme les balles ne volent pas) et réglementaires.

\section{Entrer dans la fiction}

La notion de fiction est complexe à situer, parce qu'elle se décline en différents axes. Selon Jean-Marie Schaeffer ( 1999), le principe de « feintise ludique » permet ainsi l'immersion dans un monde imaginaire, sans qu'il n'y ait confusion avec la réalité. Pour reprendre le débat proposé par Olivier Caïra (201 I : 87), définir la

\footnotetext{
"Au sujet de l'implication des fans quant au monde fictionnel d'origine, rappelons que la culture participative connaît des limites, notamment eu égard aux enjeux économiques défendus par les industries culturelles et cinématographiques, mais aussi parce que ces pratiques ne sont finalement réservées qu'au seul public des fans (François, 2009b).

${ }^{12}$ David Peyron (2016) définit la culture geek comme un « mouvement culturel [...] construit sur la base d'un stéréotype dont la forme contemporaine est née dans les années 1980. II s'agit donc du geek passionné à l'extrême, recherchant immersion et expertise totale, en particulier envers les objets technologiques, la culture numérique et les œuvres de genre ». Un geek est donc une forme de « figure idéale du passionné d'imaginaire fantastique et d'activités ludiques autour de cet imaginaire ». Les joueurs de quidditch se revendiquent de cette culture geek et certains parlent même de « sport de geek ».

${ }^{13}$ || est à noter que selon les auteurs et les théories relatives aux mondes fictionnels, l'accent est plus ou moins mis sur la convergence culturelle, le schéma narratif ou les propriétés des univers créés (pour un descriptif détaillé des différents courants, voir Sánchez, 20 I6). En amont, le quidditch profite du phénomène initial de la culture de la convergence liée à Harry Potter.
} 
fiction nécessite de prendre en compte un axe formé par deux pôles (mimétique et axiomatique) sur lequel viennent se positionner les « fictions mimétiques», les « jeux de simulation » et les « axiomatiques autonomes ». Le quidditch entre, en tant que « monde alternatif », dans la catégorie des fictions mimétiques, tout en s'appuyant sur une culture de l'imaginaire - voire une culture geek (Peyron, 2014). Dans cette continuité, Françoise Lavocat (2010) aborde la notion de fiction sous l'angle des « mondes possibles ». Pensés comme des « alternatives crédibles au monde réel », les univers fictionnels deviennent des mondes de référence pour les fans/consommateurs, facilitant ainsi les logiques d'immersion à l'œuvre lors des processus d'adaptation et de mise en vie. Par ailleurs, David Peyron (2014 : 58) note que la logique transmédiatique est intimement liée à ces constructions de mondes car elle facilite la « volonté d'approfondissement immersif et participatif », mais aussi parce que différentes activités culturelles se rejoignent, donnant lieu à un sentiment d'appartenance à une même communauté. L'univers d'Harry Potter se décline sur différents supports, que les fans connaissent et s'approprient ${ }^{14}$. Sans entrer dans les débats portant sur les notions voisines, notamment celle de cross-media qui se limiterait, à la différence du transmédia, à la redondance d'une même information sur différents supports (Di Filippo, Landais, 2017 ; Bourdaa, 2012), le quidditch moldu doit être envisagé comme une adaptation dans la logique des mondes transmédiaux (Klastrup, Tosca, 2004) : ce qui importe est le système, davantage que l'histoire, permettant de reproduire, de compléter ou d'augmenter le microcosme originel.

L'ancrage dans la fiction et la logique transmédiatique à l'œuvre autour d'Harry Potter invitent à questionner l'appropriation et la re-création du quidditch sous l'angle de la fabrique des mondes. S'appuyant sur les propositions de Tolkien pionnier en la matière - pour qui les créations fictionnelles (《 secondaires ») sont issues du monde « primaire » (notre univers « réel »), Mark Wolf développe une théorie de la construction des mondes (World-building), qui prend principalement racine dans la fantasy et la science-fiction. Ces mondes, pour être fonctionnels, reposent sur trois critères essentiels : le degré d' « invention », celui d'« intégralité » et celui de « cohérence $\gg^{15}$ (Wolf, 2012 : 33). La distance, le foisonnement de détails et l'habitabilité sont les éléments qui permettent l'autonomie des mondes fictionnels (Peyron, 2016). Ainsi, les univers imaginaires ont-ils pour particularité de regorger de descriptions pour permettre leur élaboration mais aussi pour faciliter leur « visite », impliquant une forme d'« immersion » (Wolf, 20I2) du

\footnotetext{
${ }^{14}$ À titre d'illustration, la pièce de théâtre jouée pour la première fois en juillet 2016 mettant en scène Harry Potter et l'Enfant maudit (ouvrage de J. K. Rowling se déroulant 19 ans après le dernier tome d'Harry Potter) était largement attendue par les fans, qui y voyaient une manière de se replonger dans l'univers, comme en témoigne Sébastien (26 ans) : « Et l'univers continue. Il y a une pièce qui est en train de sortir en Angleterre. Ils vont sortir le scenario en version papier, qui n'est pas un nouveau bouquin, qui n'est pas un nouveau film [mais] qui va tourner en Europe et dans le monde. II va y avoir Les Animaux fantastiques. On sait déjà qu'ils vont être en trois films. Et après, l'univers d'Harry Potter est tellement vaste que des préquelles, ou autres [ce n'est pas ce qui peut manquer]... ».

${ }^{15}$ « Invention, completeness and consistency ».
} 
lecteur. Les mondes ont nécessairement à voir avec la culture de la convergence, en ce qu'ils se diffusent d'une création individuelle à une pluralité de « visiteurs » lecteurs (ibid. : 9 ; Peyron, 2008 : 359). Plus spécifiquement, ces microcosmes sont organisés selon différents degrés de « subcréation ${ }^{16}$ » (notion qui implique autant le processus que le produit final [Wolf, 2012 : 24]), dépendants d'une superposition forte ou faible vis-à-vis du monde primaire. L'enjeu est bien, pour les créateurs de monde (world-makers), que l'univers dépasse l'histoire (Peyron, 2008), afin de prolonger l'immersion dans la fiction (Besson, 2004).

Concernant Harry Potter, le degré de subcréation est relativement élevé pour l'école de magie («Poudlard ») et ses environs. En outre, J. K. Rowling fait volontairement osciller son univers entre le monde primaire (qu'elle nomme moldu) et le monde secondaire dans lequel se déroule la majeure partie de l'histoire. Les deux espaces sont séparés par les trajets en train, symbolisant le passage d'un monde à l'autre. Parmi les éléments du décor développé par l'auteure, le quidditch - en tant que sport national des personnages sorciers - occupe une place centrale. En effet, s'il est une toile de fond permettant des avancées dans la narration, il existe aussi indépendamment de l'histoire du personnage de Potter. Des éléments « horschamps » sont proposés au lecteur lorsque, par exemple, les noms de champions illustres sont cités ou que la Coupe du monde est présentée comme un événement sportif majeur dans le monde magique. Il est ici question d'un approfondissement du microcosme sportif, qui existe en dehors même des romans et des narrations. C'est bien à travers cette « extension » du monde secondaire que le quidditch moldu a pu être mis en œuvre, comme le précise un joueur : « Quand on lit le livre, c'est vraiment une esquisse des rulebooks [livres de règles] que l'on a actuellement. Les règles sont toutes très bien détaillées, avec même un descriptif de chaque technique autorisée pendant un match. C'est tellement complet que quand on lit la chose, on se dit que ça existe vraiment! » (Alexandre, 2 I ans). Le désir de mettre en place cette activité repose, d'une part, sur la logique transmédiatique de construction des mondes et, de l'autre, sur les attentes et désirs des fans. Le souhait de prendre part à la fiction en en mettant une partie en vie traduit une attitude ludique qui n'est que le prolongement des pratiques de fanfictions. En somme, une réappropriation culturelle est à l'œuvre, forme de « braconnage » des usages (Certeau, 1990) : la consommation des livres et des films n'est plus seulement liée aux pratiques culturelles mais se retrouve également dans le domaine des activités sportives.

En dehors des logiques économiques, l'adaptation d'un monde merveilleux à une activité réelle s'inscrit dans une logique de transfictionnalité (Saint-Gelais, 2007), permettant de penser la rupture d'avec le texte ou le cinéma pour proposer une nouvelle conception du sport cher à Harry Potter. Le quidditch en tant que pratique physique conserve les éléments propres au monde sorcier imaginé par J. K. Rowling (par exemple, les noms des équipes font écho à ceux proposés dans la fiction). En

\footnotetext{
${ }^{16}$ Le terme subcréation (en anglais, subcreation), renvoie à l'idée que les mondes ne sont pas créés ex nihilo mais reposent sur une combinaison de l'existant. Ces inventions ne sont pas le fait d'êtres divins, mais bien le produit de l'imaginaire humain (Devaux, 2007).
} 
ce sens, il fait partie d'un monde transmédial (Klastrup,Tosca, 2004) : en passant d'un support (écrit) à un autre (physique), il met en jeu un degré d'expérimentation élevé. Ce qui importe, dans une logique d'adaptation ${ }^{17}$, est la manière dont est vécue la re-création et les processus d'appropriation mis en place.

\section{Le quidditch : entre immersion et appropriation}

\section{Sport sorcier versus sport « moldu»}

Au-delà de l'ancrage du quidditch dans le monde d'Harry Potter, une mise en perspective entre le sport tel qu'il est présenté par J.K. Rowling (le « sorcier ») et celui re-créé doit être faite. En effet, il n'est possible de comprendre le fonctionnement de l'activité contemporaine qu'en prenant en considération les aménagements nécessairement mis en place. II n'est pas question de dresser un historique du quidditch $\mathrm{fictif}^{18}$, mais de mettre en exergue les éléments permettant de saisir la structuration du sport moldu vis-à-vis de la fiction. Si l'imbrication des mondes se laisse deviner par certains aspects (par exemple, le vocabulaire technique issu de l'imaginaire a été conservé), l'activité re-créée se détache également de sa matrice fictionnelle en entrant dans le champ du loisir sportif.

Le mot « quidditch » - inventée par l'écrivaine - a plusieurs origines. II renvoie aux sonorités de «Yiddish », rappelant certains parallèles entre le roman et I'histoire de la Seconde Guerre mondiale (Smadja, 200 I). L'appellation du sport semble aussi formée d'après les premières lettres des noms anglais des balles: « Le Quaffle a prêté son QU, les Bludgers, leur D, et le Snitch, son ITCH. Rowling y a ajouté un I » (Deplanque, 2003 : 49). J. K. Rowling prend soin, par ailleurs, de proposer une transformation de l'orthographe qui était, au départ, Kwidditch. Avec ces précisions, elle renforce le background de ce monde secondaire. L'ouvrage spécifique présentant le quidditch commence par une préface rédigée au nom de Dumbledore, sorcier responsable de l'Académie de magie. L'objectif est de plonger le lecteur dans l'univers magique, en lui donnant une place de « moldu ». En effet, le directeur signale que l'ouvrage est dorénavant accessible aux non sorciers. II poursuit en expliquant que l'achat de ce livre servira une œuvre caritative. Ici, les mondes primaires et secondaires sont entièrement mêlés. En effet, l'œuvre caritative, britannique, existe bel et bien et les recettes issues des ventes lui sont reversées. Le lecteur est à la fois dans et hors la fiction. Le monde primaire (« réel ») est importé dans le monde secondaire (fiction).

\footnotetext{
${ }^{17}$ Isabelle Périer (2016 : 185) précise que l'adaptation, dans le cas du passage d'une fiction romanesque au monde du jeu de rôle (comprenant donc un changement de média), implique des transformations du monde fictionnel de départ. Ce phénomène traduit un attachement à l'œuvre initiale mais également un désir de nouveauté associé à la dimension ludique.

${ }^{18}$ L'analyse de cette pratique fictive a été réalisée par Samantha Deplanque (2003).
} 
Ce balancement entre les deux mondes est renforcé par le fait qu'au-delà d'un ancrage dans l'imaginaire, le quidditch fait écho à des pratiques sportives clairement identifiées. Samantha Deplanque montre qu'il est un savant mélange de sports collectifs : basket-ball, handball, rugby ${ }^{19}$, football et cricket. En outre, d'autres références culturelles renvoyant à la société anglo-saxonne sont présentes. C'est le cas du lieu de pratique : « Poudlard » est ainsi assimilé aux collèges traditionnels anglais du XIX ${ }^{e}$ siècle (Deplanque, 2003 : 6- I0). Le quidditch magique est, par ailleurs, présenté comme un sport de glisse ${ }^{20}$, les balais servant à se déplacer dans les airs. Cet aspect est perdu dans la version moldue, faute de magie et de possibilité de vol. Dès lors, les adaptations réalisées pour rendre le quidditch opérationnel dans le monde primaire passent nécessairement par un changement dans les espaces occupés. Si le balai est conservé sous forme de bâton (rappelant ainsi l'origine de l'activité et servant à organiser la pratique en termes tactiques et techniques), le quidditch devient terrestre. Il est, d'ailleurs, parfois nommé « ground quidditch ${ }^{21}$ ». Ce passage de l'air à la terre questionne le rapport des sports à l'espace, tel que François Vigneau (2008) a pu l'analyser. Prenant en compte les modalités de déplacement, le géographe note que les sports collectifs se déroulent dans un espace de type rectangulaire. Les sports de but ${ }^{22}$, dont le quidditch relève, consistent à conquérir l'espace et à s'approprier le terrain adverse. Insistant sur le « sens » donné au sport, François Vigneau avance l'idée selon laquelle le plaisir recherché dans ce type de pratique renverrait à la joie. En effet, « fondamentalement, à chaque rencontre, le sportif peut gagner, perdre ou, parfois, faire "match nul" [...]. La victoire se juge souvent sur la comparaison de la somme des points marqués par chacun des deux adversaires au cours de l'assaut ou de la partie. [lls] peuvent donc constituer autant de motifs de joie "intermédiaire" » (Vigneau, 2008 : 14). Le sport d'Harry Potter est souvent décrit comme un motif de joie par les participants car il suscite des émotions et effusions collectives (c'est le cas des « câlins 》 entre équipes, de rigueur après les matchs). Si le milieu sur lequel se pratique le quidditch change lors de son passage en version moldue (de l'air au sol), en revanche, les motifs de plaisir demeurent les mêmes que ceux mis en exergue par les personnages de fiction.

Actuellement, le quidditch moldu en est à sa $10^{\mathrm{e}}$ version des règles. Les dernières variantes visent à proposer un jeu « plus rapide et plus fluide pour les spectateurs ${ }^{23}$ », tout en mettant l'accent sur la « protection des joueurs » (Anne-Lise, 34 ans). À ce

\footnotetext{
${ }^{19}$ À ce propos, J. K. Rowling (2012, chap. 5) évoque également le vocabulaire du rugby lorsqu'elle fait référence aux « $3^{\mathrm{e}}$ mi-temps 》 d'une certaine équipe de quidditch.

${ }^{20}$ S. Deplanque (2003 : 16) note que J. K. Rowling s'appuie sur les tendances en matière d'innovation pour ancrer le quidditch dans une contemporanéité sportive.

${ }^{21}$ Le terme « ground quidditch » est notamment repris par la Fédération du quidditch français : http:// www.quidditch.fr/.

${ }^{22}$ Le quidditch (magique et moldu) s'appuie sur un terrain proche de celui du cricket. Dans la version re-créée, ses dimensions sont réduites par rapport à la pratique magique (largeur et longueur sont, environ, divisées par trois).

${ }^{23}$ ॥ existe même, traduit en français, un manuel pédagogique destiné aux batteurs, afin de leur permettre d'améliorer leur jeu (Archer, 20I5).
} 
propos, s'il n'y a pas de remplaçant prévu dans la version magique, le sport moldu permet à une équipe de proposer jusqu'à 21 joueurs (pour 7 en jeu). Globalement, le jeu réel limite l'agressivité physique par rapport au sport sorcier, même si celui-ci a lui-même connu (à travers les écrits de J. K. Rowling) un processus limitant la violence acceptable. Ainsi, la vitesse est réduite par rapport à un déplacement de sport de glisse (balai volant), impliquant des chocs moindres lors des affrontements. Les règles actuelles sont issues des États-Unis, et des différences entre quidditch nord-américain et européen se font ressentir, au niveau tant réglementaire que ludique : « II y a eu des aménagements dernièrement sur des détails de règles, dont l'exemple type est le protège-dents. Obligatoire pour les États-Unis, juste proposé et conseillé pour la fédération internationale » (Sébastien, 26 ans). L'une des règles centrales du quidditch est celle de la mixité : la pratique se présente comme le seul sport de contact mixte. Initiée dans la fiction (J. K. Rowling parle systématiquement de joueurs 《 sorciers et sorcières »), cette règle s'est renforcée lors de la re-création du quidditch. La « règle des genres » ou règle des « quatre maximum ${ }^{24}$ » est ainsi une spécificité, sur laquelle insistent les joueurs. Non seulement les équipes doivent être mixtes (hommes/femmes), mais elles doivent également prendre en compte le genre au-delà du système binaire habituellement retenu. Sur cette question, la réalité va plus loin que la fiction, dans laquelle la notion de transgenre n'apparaît pas. II s'agit bien, pour les joueurs, de prolonger certaines des valeurs véhiculées par la fiction, notamment la tolérance et la lutte contre les discriminations (Jenkins, 2009).

Les aménagements, qu'ils soient réglementaires ou en termes d'espaces, permettent la re-création du quidditch et une forme d'immersion apparaît : l'activité permet le passage dans le monde magique, même si celui-ci est transformé. En somme, le quidditch moldu permet de poursuivre l'histoire fictionnelle, sans qu'il y ait nécessairement une continuité. Ce qui importe est la représentation que les joueurs ont de l'univers qu'ils souhaitent mettre en vie. Les références à la diégèse sont présentes dans la pratique, dans ses règles, mais aussi dans ses représentations, comme les logos des associations qui se basent presque systématiquement sur des emprunts aux romans. La re-création permet ainsi, non seulement de « visiter » un monde (Wolf, 20 I2) mais aussi de participer à la construction d'un nouvel univers.

\footnotetext{
${ }^{24}$ Les règles officielles traduites en français ( $8^{e}$ édition) exposent ce point : « Lors d'un match de quidditch, une équipe peut compter en même temps sur le terrain au maximum quatre joueurs actifs, sans compter l'attrapeur, qui s'identifient au même genre. Le genre avec lequel un joueur s'identifie est considéré comme étant le genre de ce joueur, et qui peut être, ou non, le même que le sexe de cette personne. On fait communément référence à cette règle sous le nom de règle des "quatre maximum". L'use [fédération du quidditch aux Étast-Unis] accepte les personnes qui ne s'identifient pas au sein du système de genre binaire et reconnaît que tous nos joueurs ne s'identifient pas comme étant homme ou femme. L'use accueille des personnes de tous genres et identités dans notre ligue » (Us Quidditch Rulebook. Version française, 2014 : I0. Accès : http://angersquidditch.fr/docs/Reglement8-I.pdf).
} 


\section{D'un monde à l'autre}

Si le monde d'Harry Potter est une fiction, J. K. Rowling prend toutefois soin d'« inverser les rapports entre le monde réel et le monde imaginaire : elle fait comprendre que le monde le plus proche de la réalité n'est pas celui des Dursley [moldus] mais bien celui de Poudlard et de ses sorciers » (Smadja, 200 I : II). L'enjeu majeur est de rendre tangible le monde imaginaire, de lui donner une consistance et, ainsi, un « effet de vie » (ibid.). Si les romans mettent tout en œuvre pour produire un imaginaire « réel », la re-création du quidditch interroge l'imbrication des univers à un autre niveau.

D'abord, l'activité sportive s'appuie clairement sur le monde imaginaire afin de donner naissance à une pratique innovante. Le processus est inversé : ce n'est plus la fiction qui se fonde sur des éléments de la réalité (Jenkins, Wolf, 20 I3), mais le contraire. Le « monde primaire », autrement dit, le sport réel, s'appuie sur des données de l'imaginaire. Dès lors, le quidditch moldu ne serait-il pas le monde secondaire du monde secondaire ? Son degré de « secondariness ${ }^{25}$ » (Wolf, 2012) est proche, non pas du monde primaire (dans lequel le quidditch n'existe pas) mais bien d'un microcosme inventé. En ce sens, le sport moldu parait être la subcréation d'un imaginaire. Cette conception du quidditch permet d'expliquer l'attrait qu'il peut avoir auprès des joueurs : il ne s'agit plus seulement de prolonger le monde, mais de créer une sorte de mise en abîme de l'univers imaginaire. II n'est pas uniquement question de reproduire le sport d'Harry Potter mais aussi de l'ancrer dans une logique de fabrique d'un monde à part entière, puisque les matrices peuvent exister sans les histoires (Wolf, 20 I2).

D'ailleurs, certains codes et valeurs véhiculés dans la narration sont réappropriés par les lecteurs. L'engagement militant des consommateurs de cette fiction aux États-Unis illustre bien ce phénomène. La génération des « Millenials ${ }^{26}$ » exprime des tendances politiques semblables aux valeurs défendues dans les romans par le personnage de Harry (par exemple, ne pas juger les gens sur leur apparence ou leur appartenance sociale, reconnaitre des droits égaux à chacun, proscrire la violence, etc.) (Gierzynski, 20।3). Cette population reproduit les leçons apprises par les personnages des romans. On retrouve ici les critères constituant les mondes transmédiaux, en particulier l'« ethos ${ }^{27}$ » (Klastrup, Tosca, 2004). Celui-ci, organisant l'éthique du monde et les valeurs morales qui en font partie, expose

${ }^{25}$ M. Wolf $(2012: 26)$ définit ainsi le principe de secondariness : « Cela dépend du niveau de détachement et de différence entre l'endroit décrit et le monde Primaire, et du degré à partir duquel ses aspects fictionnels ont été développés et construits » (« [It] dépends on the extent to which a place is detached from the Primary World and different from it, and the degree to which its fictional aspects have been developed and built »).

${ }^{26}$ A. Gierzynski reprend le terme « Millenials » à Neil Howe et Bill Strass : il désigne les enfants nés entre 1982 et le milieu des années 90.

${ }^{27}$ Les deux autres éléments qui composent les mondes transmédiaux sont les suivants : « Mythos », qui organise l'histoire du monde et «Topos » qui fournit le « cadre géographique » (Di Filippo, 20 I7). 
également les lignes de conduites des personnages. Dans le cas du quidditch, les pratiquants s'attachent à suivre les valeurs défendues par Harry Potter. Au-delà des répercussions sur l'engagement politique, le débordement de la fiction dans les manières de concevoir le monde se retrouve chez les quiddkids : « II ne faut pas se leurrer, ces livres nous apprennent quand même la vie... Toute cette génération qui a été bercée dans Harry Potter a une façon de penser qui est totalement en marge de ce que les gens pensent en général » (Alexandre, $2 \mathrm{l}$ ans). Une dimension éducative ou, tout du moins, une fonction socialisatrice de l'œuvre se laisse deviner, conduisant les fans à se regrouper autour d'une activité. Les conséquences que la lecture a pu avoir sur la construction de l'identité individuelle et la recherche d'une sociabilité permettant l'expression de valeurs intériorisées dans la jeunesse expliquent l'engouement pour la mise en vie de l'univers.

Un processus de fictionnalisation est à l'œuvre puisque le réel reproduit la fiction : «Les cas de fictionnalisation (de mise en fonction donc) du réel [...] correspondent à une véritable révolution dès lors que ce n'est plus la fiction qui imite le réel mais, semble-t-il, le réel qui reproduit la fiction » (Augé, 1997a : I I 5). Le passage d'un sport fictif à un jeu réel illustre parfaitement ce renversement. Dans les romans, le quidditch est un sport. Dans la réalité, la re-création se conçoit, en Europe, avant tout comme un jeu. Si le terme de « sport » est de plus en plus utilisé (en partie suite à l'internationalisation des rencontres), l'accent est mis, par les joueurs, sur la dimension d'innovation. Le quidditch est vécu comme un sport «non traditionnel», un sport « alternatif » (Jarvie, 2006), dont les valeurs font écho au « fun », aux cultures de genre ${ }^{28}$, à la dimension ludique au détriment d'une approche agonistique, etc. (Cohen, Peachey, 2014 : 522). C'est ce que note Raphaël : « Le quidditch s'est beaucoup transformé... Même les Américains se posent la question : est-ce qu'on ne va pas vers trop de sport? Et on oublie ce qu'était le quidditch à la base : un jeu » (Raphaël, 26 ans).

Ensuite, cette création d'un univers non magique qui enclenche des expériences à vivre et qui regroupe des acteurs autour d'un objectif commun (jouer à un sport issu de la fiction) peut inviter à concevoir le quidditch français comme un monde social, au sens que Howard Becker (1982) donne à ce terme ${ }^{29}$. Cette approche peut permettre de comprendre le « monde du quidditch » sous l'angle de structures d'activités collectives, tout en mettant au centre les logiques d'action

\footnotetext{
${ }^{28}$ Les auteurs parlent ici de « sub-cultures », que l'on pourrait traduire par « sous-cultures » ou, dans ce contexte, par « cultures de genre ».

${ }^{29} \mathrm{H}$. Becker évoque le « monde » comme étant une « métaphore », qui « contient des gens, toutes sortes de gens, qui sont en train de faire quelque chose qui leur demande de prêter attention les uns aux autres, de tenir compte consciemment de l'existence des autres et de donner forme à ce qu'ils font en conséquence [...]. Ils développent graduellement leurs lignes d'activité » (Becker et Pessin, 2006 : 168). Ailleurs, H. Becker (1982 : 58-59) propose de comprendre le monde (de l'Art) comme étant composé de « toutes les personnes dont les activités sont nécessaires à la production des œuvres bien particulières [...]. [C'est] un réseau de chaînes de coopération qui relient les participants selon un ordre établi ».
} 
des acteurs. Bien qu'il ne s'agisse pas d'un « monde » organisé autour d'une activité professionnelle, envisager le quidditch à l'aune de « conventions » et de « chaînes de coopération » invite à saisir l'importance des valeurs partagées et les lignes de démarcations de l'activité. II serait ainsi, comme d'autres pratiques ludiques, un « monde du jeu ${ }^{30} »$ (Berry, Borzakian, 20I5). Malgré deux tendances fortes et souvent opposées (ceux qui sont présents car ils recherchent une nouvelle activité sportive et ceux qui viennent au quidditch parce qu'ils sont fans de la fiction), les joueurs coopèrent entre eux et l'activité se développe, sur un plan tant quantitatif (augmentation régulière du nombre de joueurs depuis cinq ans) que structurel (création de la fédération en 2012, organisation de trois Coupes de France, etc.). Même si des dissensions existent au niveau institutionnel et, parfois, entre clubs et instances dirigeantes (notamment sur les objectifs poursuivis par la fédération), il n'en reste pas moins que des consensus sont réalisés, des conventions respectées (en particulier concernant les attentes en termes de développement de l'activité) et des tâches assignées à chacun (ce sont, par exemple, les différentes commissions de la fédération ou les groupes de travail, mais aussi au niveau local, le « responsable » de la création des balais, etc.). L'objectif est de permettre, non seulement la progression du quidditch en France (et en Europe), mais aussi l'amélioration du niveau (pour réaliser des performances sportives lors des différents championnats). Cette grille de lecture permet d'expliquer pourquoi les tensions en jeu au sein de l'activité ne conduisent pas à son éclatement : à l'heure actuelle, les acteurs sont en train de construire leur pratique, de la positionner dans l'espace des sports (Pociello, 1995), de créer « une démarcation entre ce qu'ils veulent donner pour caractéristique et tout le reste » (Becker, 1982 : 60).

\section{Une communauté de pratique?}

Dans la logique de construction de mondes, la re-création est une appropriation. Pour ce qui est du quidditch, les nécessaires adaptations ont permis de donner naissance à une activité nouvelle, qui oscille entre ancrage dans un monde secondaire (en particulier avec les joueurs fans) et un monde social, permettant à la pratique de progresser. Cette conception de l'activité comme structure permet de faire le lien entre le processus de world-building et la dimension communautaire. Au-delà d'un monde social, le quidditch peut aussi être appréhendé comme une « communauté de pratique » (Wenger, 2005). En tant que lieu d'apprentissage, une communauté de pratique "se définit à la fois par un "répertoire partagé" entre les membres, un "engagement mutuel" de ces derniers dans la pratique et une "entreprise commune" visant à les réunir » (Brody, 2015 : 2). Les associations de quidditch sont encore peu nombreuses en France. De fait, le groupe fonctionne

\footnotetext{
${ }^{30}$ Dans leur article,V. Berry et M. Borzakian (2015) montrent que la notion de monde du jeu « permet d'évoquer la chaîne des agents qui participent de la production d'une activité ludique et d'un univers culturel spécifique [...]. Parler de monde du jeu à propos d'une activité ludique permet ainsi de porter une attention particulière à la diversité des acteurs qui contribuent à son existence ».
} 
selon un niveau d'interconnaissance restreint (nombreux échanges numériques, rencontres sportives fréquentes, etc.), parce qu'il est, pour le moment, réduit. L'apprentissage au contact des autres est l'un des éléments fondamentaux mis en avant par les joueurs. Au-delà de l'activité physique, se laisse deviner une communauté soudée autour de valeurs communes.

En premier lieu, ce qui rassemble les joueurs de quidditch est une connaissance partagée du monde de «Poudlard », en général, et du quidditch, en particulier. Chaque membre du groupe est ainsi doté d'une forme de capital culturel lui permettant d'afficher une expertise (Peyron, 2008) relative au monde d'Harry Potter. Comme le personnage du jeune sorcier, les joueurs revendiquent, par exemple, des désirs d'aventure et de dépaysement ${ }^{31}$ : le merveilleux de la fiction est transposé dans les déplacements. C'est ce que rappelle Raphaël : « [Ce que j'aime dans le quidditch], c'est voyager. Vu qu'il y a peu d'équipes, on est amené à aller un peu partout. Vu que l'aspect communauté est présent, cela permet de rencontrer des gens dans d'autres pays avec qui, après, on garde des contacts » (Raphaël, 26 ans). Les voyages, les rencontres et l'altérité forment le cœur du répertoire sur lequel repose la communauté de pratique. Ces attentes ne sont, d'ailleurs, pas éloignées des messages véhiculés par les contes de fées ${ }^{32}$ (Propp, 1928 ; Fournier, $2013^{33}$; Smadja, 200 I). Ce lien à l'imaginaire est renforcé par les signes de reconnaissance portés par les individus, notamment les plus grands fans : écharpe aux couleurs de la « maison » d'Harry Potter, romans présents dans les sacs de sports, etc. II s'agit de s'inscrire dans la continuité du monde imaginaire et de montrer son adhésion à la fiction. Par ailleurs, un fort sentiment d'appartenance et une reconnaissance entre pairs sont exprimés par les joueurs : « Le fait que quelqu'un joue au quidditch, ça te dit quelque chose sur cette personne... » (Milena, 19 ans) ; « Quand tu fais du quidditch [...], tu cernes beaucoup mieux les gens. Très souvent, les gens qui vont dans le quidditch se ressemblent tous un petit peu. Ils ont des passions communes, ils peuvent enfin appartenir à [quelque chose qui leur ressemble]... » (Sarah, 2 I ans). La communauté se soude autour des valeurs d'entraide et de soutien, au sein des équipes mais aussi entre elles : «Ce que j'adore dans le quidditch, c'est l'esprit d'équipe. Et aussi, que cela soit mixte. Parce que franchement, j'ai fait des sports en communauté, mais on était que entre gars [...]. Alors que dans le quidditch, c'est mixte, c'est plus cool. Tu as un autre esprit » (René, 17 ans). Les règles officielles exposent aussi clairement la dimension communautaire. La fédération des États-Unis, qui rédige le règlement, a fait apparaître la « règle des $3 C$ » : « Ils représentent les trois piliers du travail de l'USQ : compétition, communauté, et créativité ${ }^{4}$ ». Sous l'item « communauté », est rappelée la mixité du sport, mais sont également mis en avant les événementiels pour

\footnotetext{
${ }^{31}$ Ce va-et-vient entre dépaysement et monde réel est également effectif lors de la lecture des romans (Smadja, 2007).

32 À ce propos, Harry Potter pourrait entrer dans la catégorie fairy-tale fantasy, qui prend ses origines dans les contes traditionnels (Baudou, 2005).

${ }_{33}$ M. Fournier s'attache, en particulier, à faire le lien entre littérature jeunesse, expérience fictionnelle et voyage dans un univers imaginaire.

${ }^{34}$ us Quidditch Rulebook. Version française, 2014 : 9. Accès : http://angers-quidditch.fr/docs/Reglement8-I.pdf.
} 
faire connaître l'activité, l'apprentissage aux enfants et l'ouverture culturelle. Cette revendication d'appartenance se double d'un engagement mutuel : chaque équipe fait partie d'une plus large communauté et chacune se sent responsable des autres, au niveau local, national ou international. À titre d'exemple, Sébastien raconte un temps fort de la préparation de la dernière Coupe du monde : « On suit certaines équipes [pour la coupe du Monde] sur les réseaux sociaux, genre l'Ouganda. Certaines équipes africaines galèrent pour venir. Elles ont fait un crowdfunding [pour que les membres des autres équipes à l'international les aident]. Si les joueurs arrivent à venir, ce serait vraiment super! » (Sébastien, 25 ans).

À travers l'apprentissage des valeurs associées au quidditch (dont certaines sont directement issues de la fiction), l'entreprise commune prend son sens : le but est le développement de cette pratique « alternative ». En partageant une connaissance et un attrait pour le monde magique créé par J. K. Rowling, les quiddkids valident tacitement un engagement mutuel visant à structurer, normaliser et médiatiser le quidditch moldu. Même si des dissensions sont présentes et connues au sein des équipes ou entre les équipes, ce qui prime est la cohésion du groupe, qui repose sur un attachement commun au monde fictionnel et sur une pratique « originale » : « Les compétitivités, ça fout un peu le bordel ! [Rires]. Mais sinon, ce qui importe est le fait de partager quelque chose que tout le monde ne connait pas forcément ou ne comprend pas forcément » (Benjamin, 25 ans).

\section{Conclusion}

L'intérêt porté par les sciences humaines et sociales aux mondes imaginaires n'est pas récent et trouve un point d'ancrage fort en ethnologie, l'accent pouvant être mis, par exemple, sur la compréhension d'imaginaires collectifs (Augé, 1997a et b) mais aussi sur les enjeux des usages de cette notion (Colleyn, 2005). Comprendre les univers fictionnels a, depuis longtemps, été un objectif afin de saisir des manières d'être au monde. Entre logiques mimétiques, représentationnelles, ou encore, narratives, les aménagements du réel questionnent une forme d'invariant anthropologique. La re-création du quidditch permet de comprendre comment un phénomène culturel et social tel que les romans et films Harry Potter, a pu donner lieu à un monde qui est non seulement consommé mais aussi investi par les fans. Un double mouvement est à l'œuvre autorisant la réalisation d'un sport, au départ fictionnel. La théorie de la convergence culturelle - notamment selon Henry Jenkins - expose l'engouement pour l'univers sorcier et la création de communautés de fans. Parallèlement, le système des mondes transmédiaux - tel que l'abordent Lisbeth Klastrup et Susana Tosca - fait le lien avec la mise en vie du quidditch. Si ce dernier ne s'intègre pas directement dans la narration, il est cependant une adaptation du monde et ses pratiquants continuent à diffuser l'« ethos » de celui-ci, à travers les valeurs qu'ils défendent. La fiction entre dans la réalité, le réel complète et augmente le merveilleux. II ne s'agit pas simplement 
de rompre avec l'imaginaire pour donner naissance à une pratique sportive contemporaine. Au contraire, les logiques d'action des matrices immersives sont toujours à l'œuvre dans le quidditch « terrestre ». C'est seulement en comprenant, d'une part, le processus de world-building et la création de mondes secondaires et, de l'autre, les logiques de conventions ou de revendications communautaires que les enjeux et les objectifs du sport sorcier re-créé peuvent être saisis.

En outre, le quidditch ne constitue qu'une seule facette de l'univers d'Harry Potter : les fanfictions, entre autres, en font aussi partie. Dès lors, la pratique sportive vient s'ajouter à ces autres dimensions qui fabriquent des «mondes transmédiaux». Chaque fragment participe à la création d'un imaginaire plus vaste et se positionne dans une « logique de co-construction des mondes ». En somme, « chaque élargissement du virtuel retentit sur la complexité des différents mondes et chaque monde participe vraisemblablement à l'invention de nouvelles virtualités » (Petitat, 2006 : 106). Olivier Caïra (201 I : 39-54) insiste, par ailleurs, sur l'importance des « versions », constitutives des microcosmes. Les cadres de la fiction ne peuvent ainsi se comprendre qu'en lien avec une « concordance des versions », induisant une expérience qui soit structurée. L'approche sociologique de l'activité physique re-créée permet de faire état des connexions de l'imaginaire au monde « primaire ». Le monde secondaire apparait comme un élément constitutif de la communauté des joueurs. Ceux-ci savent qu'ils ne se positionnent pas dans le merveilleux mais ce qu'ils mettent en place est une expérience qui reprend certains éléments de l'univers magique. Olivier Caïra (ibid. : 175) évoque la nécessité de n'avoir qu'un « seul monde » pour traiter les théories de la fiction. lci, l'imbrication des matrices semble suivre cette conception, le quidditch moldu pouvant être compris comme le monde secondaire d'un autre monde secondaire, plus vaste.

II est enfin possible de rappeler que la littérature de l'imaginaire séduit également parce qu'elle permet une forme d'évasion en dehors du réel. Dès lors, l'apparition du quidditch moldu ne pourrait-elle être comprise comme une réponse à un désir de transformer un quotidien monotone ? Plus encore, la génération qui a grandi avec Harry Potter ne cherche-t-elle pas, selon ce modèle, à réenchanter le monde ? Le quidditch moldu serait ainsi une réponse à une angoisse d'une société figée et viserait à réinstaurer une part de merveilleux dans le quotidien : «Ce genre de personne [les fans d'Harry Potter] est toujours considérée comme un bisounours. On essaie de voir le positif là où il n'y en a pas. On est tous un peu comme ça. Et vu que l'on se ressemble tous, on s'aime tous, on se fait tous des câlins! » (Alexandre, 2 I ans).

\section{Références}

Archer N., 20 I5, L'Art du cognard. Un guide pédagogique de Quidditch, trad. de l'anglais par N. Esmein, s.l., Amazon.

Augé M., 1997a, « De l'imaginaire au "tout fictionnel" », Recherches en communication, 7 , pp. $105-120$. 
Augé M., 1997b, L'Impossible voyage. Le tourisme et ses images, Paris, Payot.

Baudou J., 2005, La Fantasy, Paris, Presses universitaires de France.

Becker H., 1982, Les Mondes de l'art, trad. de l'anglais par J. Bouniort, Paris, Flammarion, 2010.

Becker H., Pessin A., 2006, « Dialogue sur les notions de Monde et de Champ », Sociologie de l'Art, 8, I, pp. I63-180.

Berry V., Borzakian M., 20I 5, « Introduction. Les Mondes du jeu », Reset, 4. Accès : http:// reset.revues.org/541.

Besson A., 2004, D'Asimov à Tolkien. Cycles et séries dans la littérature de genre, Paris, CNRs Éd.

Bourdaa M., 2012, « Le transmédia : entre narration augmentée et logiques immersives », Inaglobal. Accès : http://www.inaglobal.fr/numerique/article/ le-transmedia-entre-narration-augmentee-et-logiques-immersives.

Brody A., 20I5, « Les joueurs amateurs de poker: une communauté de pratique? », Reset, 4. Accès : http://reset.revues.org/5I5.

Caïra O., 20 I I, Définir la fiction. Du roman au jeu d'échecs, Paris, Éd. de l'École des hautes études en sciences sociales.

Certeau M. de, 1990, L'Invention du quotidien. Tome I : Arts de faire, Paris, Gallimard.

Cohen A., Peachey J.-W., 2014, « Quidditch: Impacting and Benefiting Participants in a Non-Fictionnal Manner », Journal of Sport and Social Issues, 39, 6, pp. 52I-544.

Colleyn J-P., 2005, « Fiction et fictions en anthropologie », L'Homme, I 75- I 76, 3, pp. I 47- I 63.

Deplanque S., 2003, La Création d'un jeu imaginaire. Le Quidditch dans Harry Potter, Mémoire de DESS en sciences du jeu, Université Paris 13.

Devaux M., 2007, « Leibniz et Tolkien : monde possible et subcréation », pp. I55- I 68, in : Besson A., White-Le Goff M., dirs, Fantasy, le merveilleux médiéval aujourd'hui, Paris, Bragelonne.

Di Filippo L., 2017, « Conan entre personnage transmédia, monde transmédial et réalisations locales », Revue française des sciences de l'information et de la communication, 10. Accès : https://rfsic.revues.org/2578.

Di Filippo L., Landais É., 20 I7, « Dispositifs transmédiatiques, convergences et publics : construire et penser les relations entre médias », pp. 7-45, in : Di Filippo L., Landais É., dirs, Penser les relations entre médias. Dispositifs transmédiatiques, convergences, constructions des publics.

Donnat O., 2004, « Les univers culturels des Français », Sociologie et sociétés, 36, I, pp. 87- 103.

Fournier M., 2013, « Le "pays des contes". La littérature pour la jeunesse et les frontières de la fiction », Poétique, 173, I, pp. 87-104.

François S., 2009a, « Fanf(r)ictions. Tensions identitaires et relationnelles chez les auteurs de récits de fans », Réseaux, I53, I, pp. I57- 189.

François S., 2009b, « La participation médiatique selon Henry Jenkins (note critique) », Terrains \& travaux, I5, I, pp. 213-224.

Gierzynski A., 20I3, Harry Potter and the Millenials, Baltimore, The Johns Hopkins University Press. Koninck G. de, 200I, « Le phénomène Harry Potter », Québec français, I20, pp. 44-47. 
Guttmann A., 1978, Du rituel au record : la nature des sports modernes, trad. de l'anglais par T. Terret, Paris, Éd. L'Harmattan, 2006.

Jarvie G., 2006, Sport, Culture and Society: an Introduction, Londres/New-York, Routledge.

Jenkins H., 2006, La Culture de la convergence : des médias au transmédia, trad. de l'anglais par C. Jaquet, Paris, A. Colin, 2013.

Jenkins H., 2009, « How "Dumbledore's Army" is transforming our world: an interview with the HP Alliance's Andrew Slack (Part one \& Part Two) », Blog de Henry Jenkins. Accès : http://henryjenkins.org/2009/07/how_dumbledores_army_is_transf_l.html.

Jenkins H., Wolf M., 2013, « Building imaginary Worlds: An interview with Mark J. P.Wolf (Part one) », Blog de Henry Jenkins. Accès : http://henryjenkins.org/2013/09/buildingimaginary-worlds-an-interview-with-mark-j-p-wolf-part-one.html.

Klastrup L., Tosca S., 2004, «Transmedial Worlds. Rethinking Cyberworld Design », in : IEEE Computer Society, dir., Proceedings of the 2004 International Conference on Cyberworlds. Accès : http://www.itu.dk/people/klastrup/klastruptosca_transworlds.pdf.

Lavocat F., dir., 20 I0, La Théorie littéraire des mondes possibles, Paris, CNRS Éd.

Périer I., 2016, « De la fiction romanesque au monde ludique », pp. 185-200, in : Besson A., Prince N. et Bazin L., dirs, Mondes fictionnels, mondes numériques, mondes possibles, Rennes, Presses universitaires de Rennes.

Petitat A., 2006, « Fiction, pluralité des mondes et interprétation », A contrario, 4, 2, pp. 85- 107.

Peyron D., 2008, «Quand les œuvres deviennent des mondes. Une réflexion sur la culture de genre contemporaine à partir du concept de convergence culturelle », Réseaux, |48-| 49, 2, pp. 335-368.

Peyron D., 20|4, « Les mondes transmédiatiques, un enjeu identitaire de la culture geek », Les Enjeux de l'information et de la communication, I5, 2, pp. 5 I-5I.

Peyron D., 2016, « L'appropriation ludique des mondes fictionnels chez les fans d'imaginaire fantastique », ilnterrogations?, 23. Accès : http://www.revue-interrogations. org/L-appropriation-ludique-des-mondes.

Pociello C., 1995, Les Cultures sportives. Pratiques, représentations et mythes sportifs, Paris, Presses universitaires de France.

Propp V., 1928, Morphologie du conte, trad. du russe par C. Ligny, Paris, Éd. Le Seuil, 1970.

Raoul N., 2007, « Ce que Harry Potter a changé dans l'édition. Analyse et mise en perspective de "l'effet HP" dans le monde de l'édition », pp. I59-180, in : Smadja I., Bruno P., dirs, Harry Potter, Ange ou démon?, Paris, Presses universitaires de France.

Rowling J. K., 1997, Harry Potter à l'école des sorciers, trad. de l'anglais par J.-F. Ménard, Paris, Gallimard Jeunesse, 1998.

Rowling J. K., 1998, Harry Potter et la Chambre des secrets, trad. de l'anglais par J.-F. Ménard, Paris, Gallimard Jeunesse, 1999.

Rowling J. K., 1999, Harry Potter et le Prisonnier d'Azkaban, trad. de l'anglais par J.-F. Ménard, Paris, Gallimard Jeunesse.

Rowling J. K., 2000, Harry Potter et la Coupe de feu, trad. de l'anglais par J.-F. Ménard, Paris, Gallimard Jeunesse.

Rowling J. K., 200 Ia, Quidditch Through the Ages, London, Bloomsbury. 
Rowling J. K., 200 Ib, Le Quidditch à travers les âges, trad. de l'anglais par J-F. Ménard, s.l., Pottermore, 2012.

Rowling J. K., 2003, Harry Potter et l'Ordre du phénix, trad. de l'anglais par J.-F. Ménard, Paris, Gallimard Jeunesse.

Rowling J. K., 2005, Harry Potter et le Prince de sang-mêlé, trad. de l'anglais par J.-F. Ménard, Paris, Gallimard Jeunesse.

Rowling J. K., 2007, Harry Potter et les Reliques de la Mort, trad. de l'anglais par J.-F. Ménard, Paris, Gallimard Jeunesse.

Rowling J. K., 2016, Harry Potter et l'Enfant maudit, trad. de l'anglais par J.-F. Ménard, Paris, Gallimard Jeunesse.

Saint-Gelais R., 2007, « Contours de la transfictionnalité », pp. 5-25, in : Audet R., SaintGelais R., dirs, La Fiction, suites et variations, Québec, Éd. Nota Bene.

Sánchez N.-R., 20 16, « Mundos transmediales: revisión conceptual y perspectivas teóricas del arte de crear mundos », Icono 14, I4, pp. 49-70.

Schaeffer J-M., 1999, Pourquoi la fiction?, Paris, Éd. Le Seuil.

Smadja I., 200 I, Harry Potter, les raisons d'un succès, Paris, Presses universitaires de France.

Smadja I., 2007, « Harry Potter, ou la nécessité d'une approche plurielle », pp. I5-32, in : Smadja I., Bruno P., dirs, Harry Potter, Ange ou démon?, Paris, Presses universitaires de France.

Tuaillon Demésy A., 20 I 6, « Réflexions épistémologiques autour de la (re)création du geste technique de combats anciens à partir de sources historiques », Revue suisse d'histoire/ Itinéra, 39, I, pp. 21 -33.

Vigneau F., 2008, « Le "sens" du sport : conquête de l'espace, quête du plaisir », Annales de géographie, 662, 4, pp. 3-19.

Wenger É., 2005, La Théorie des communautés de pratique. Apprentissage, sens et identité, trad. de l'anglais par F. Gervais, Québec, Presses de l'Université Laval.

Wolf M., 2012, Building Imaginary Worlds. The Theory and History of Subcreation, New-York \& London, Routledge. 